\title{
Noma neonatorum in a four month old baby with pseudomonas sepsis
}

\section{Biplab Maji ${ }^{1}$}

Sri Lanka Journal of Child Health, 2014; 43(2): 110-111

(Key words: Noma; cancrum oris; noma neonatorum)

Noma or cancrum oris is an acute gangrenous stomatitis of oral and para-oral structures in the age group 2 to 16 years particularly in sub-Saharan Africa and Asia ${ }^{1}$. It has a high morbidity and mortality and is commonly caused by borellia vincentie, bacillus fusiformis, bacteroids and staphylococcal species ${ }^{2}$. Ghosal et al. from Calcutta reported 48 cases of noma and coined the term 'noma neonatorum' for an entity, seen within the first 4 months of life where pseudomonas aeruginosa is the most common causative organism $^{3,4}$.

\section{Case report}

A 4 month old male infant presented with highgrade fever for 5 days accompanied by lethargy, recurrent bouts of cough and rhinorrhoea for 3 days. He was born at term from a non consanguineous marriage with a birth weight of $2.6 \mathrm{~kg}$. He was exclusively breast fed and was immunised properly. The postnatal period was uneventful except for two episodes of pneumonia requiring hospital admission.

On examination, he had normal vital parameters with weight and height above the $95^{\text {th }}$ percentile. Mild pallor was noted. Bilateral purulent rhinorrhoea with erythema of nasal septum and turbinate were present. Respiratory examination revealed bilateral crepitations. $\mathrm{He}$ had firm hepatomegaly $(4 \mathrm{~cm}$ with span of $7.5 \mathrm{~cm}$ ) and splenomegaly $(1.5 \mathrm{~cm})$. Meningeal signs and focal neurological deficits were absent. After 48 hours of admission, he developed a rapidly progressive gangrenous patch on the tip of the nose with surrounding cellulitis (figure 1). The nasal gangrene sloughed off in one week (figure 2).

Investigations revealed leucocytosis (cell count of $19,200 / \mathrm{cu} \mathrm{mm}$ ), raised ESR (38 $\mathrm{mm}$ in $1^{\text {st }}$ hour) high C-reactive protein of 38.9 and normal liver and renal function tests. Blood culture and nasal swab showed heavy growth of Pseudomonas

${ }^{1}$ Postgraduate Trainee in Paediatrics, Institute of Child Health, Kolkata 700017, India

(Received on 22 April 2013: Accepted after revision on 21 June 2013)

aeruginosa within 48 hours of incubation. Pseudomonas isolated from the blood culture was susceptible to ceftazidime, piperacillin, gentamycin, amikacin and ciprofloxacin. Serological tests for syphilis and hepatitis B were negative. ELISA test for HIV - antibody was negative.
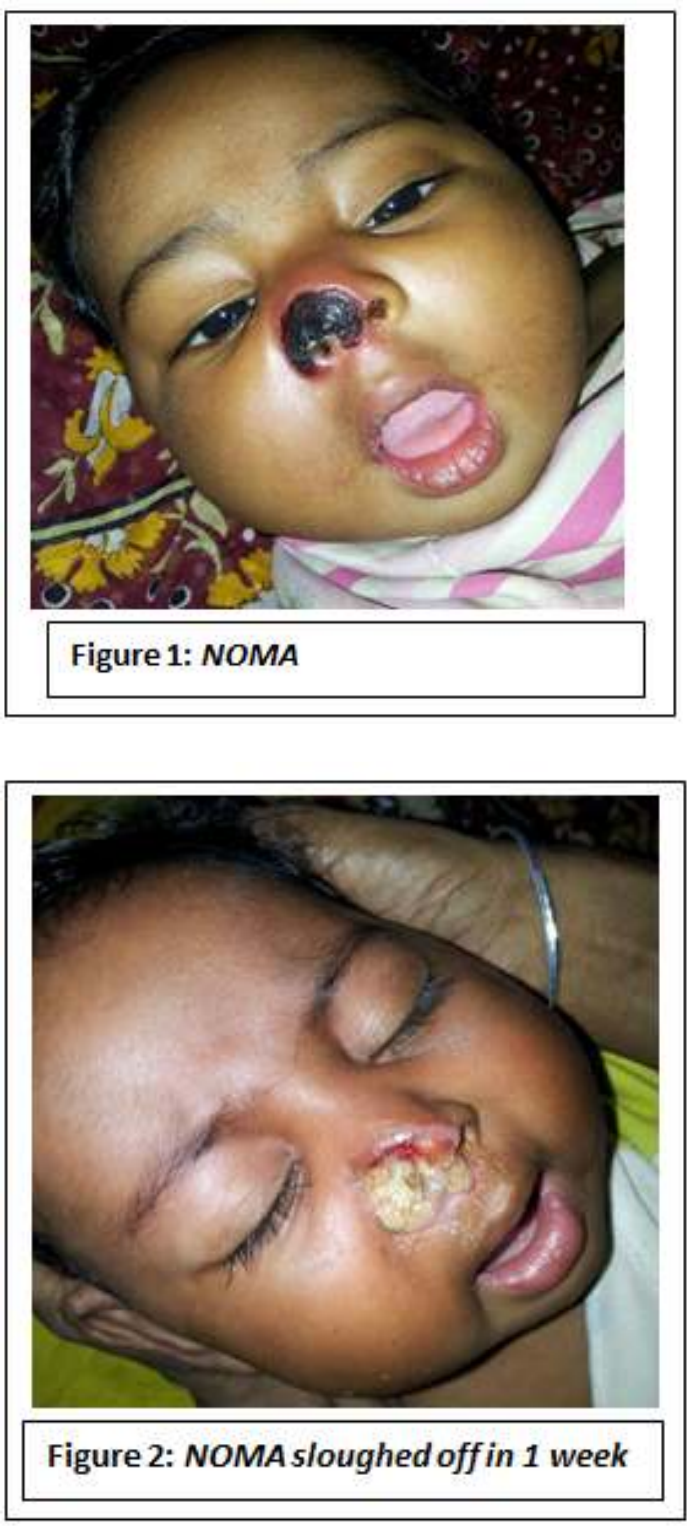
Serum immunoglobulin levels were normal. Protein C, protein S, anti-thrombin III and factor V Leiden were all within normal levels. Chest radiograph revealed bilateral bronchopneumonia. Nasal septal biopsy showed panniculitis. The patient was treated with a 2-week course of intravenous ceftazidime and amikacin. Plastic surgical nasal reconstruction was advised on discharge.

\section{Discussion}

Noma neonatorum has no predilection for birth weight or gestation. Oral cavity is the most common site of involvement, the others being nose, eyelid, umbilicus, scrotum and groin ${ }^{3}$. Onset of the disease has been reported to range from 3rd postnatal day to 120 days $^{3}$. Pseudomonas aeruginosa has consistently been isolated from all cases $^{3}$. The disease is fatal in majority of the cases, with only 6 survivors amongst 54 cases reported up to date ${ }^{3}$.

Noma neonatorum needs to be treated aggressively with antibiotic cover against Pseudomonas species. Apart from supportive care, local measures such as repeated irrigation of the local lesions seem beneficial as the pooled secretions have a heavy concentration of causative organisms and the concentration of antibiotics in the secretions attained by systemic administration is doubtful.
Extensive surgical debridement is contraindicated in these cases and reconstructive surgery is advocated at least one year after the resolution.

\section{References}

1. International No-Noma Federation (2010) Illness: introduction. Available: http://www.nonoma.org/. Accessed 29 November 2010.

2. Enwonwu CO, Falkler WA, Jr., Phillips RS. Noma (cancrum oris). Lancet 2006; 368: 147 56.

http://dx.doi.org/10.1016/S01406736(06)6900 $\underline{4-1}$

3. Ghosal SP, Chaudhari M, Dutta N,Sarkar AK, Mukherjee AK, Sen Gupta PC. Noma Neonatorum. Indian Pediatrics 1977; 14: 70914.

4. Ghosal SP, Sen Gupta PC, Mukherjee AK, Choudhury M, Datta N, Sarkar AK. Noma neonatorum: It's aetio-pathogenesis. Lancet 1978; 2: 289-90.

http://dx.doi.org/10.1016/S01406736(78)9169

$\underline{1-4}$ 